\title{
Experiments on Metamaterials with Negative Effective Static Compressibility
}

\author{
Jingyuan Qu, ${ }^{1,2, *}$ Alexander Gerber, ${ }^{2}$ Frederik Mayer, ${ }^{1,2}$ Muamer Kadic, ${ }^{2,3}$ and Martin Wegener ${ }^{1,2}$ \\ ${ }^{1}$ Institute of Nanotechnology, Karlsruhe Institute of Technology (KIT), 76021 Karlsruhe, Germany \\ ${ }^{2}$ Institute of Applied Physics, Karlsruhe Institute of Technology (KIT), 76128 Karlsruhe, Germany \\ ${ }^{3}$ Institut FEMTO-ST, UMR 6174, CNRS, Université de Bourgogne Franche-Comté, \\ 25000 Besançon, France
}

(Received 6 September 2017; revised manuscript received 27 October 2017; published 8 December 2017)

\begin{abstract}
The volume of ordinary materials decreases in response to a pressure increase exerted by a surrounding gas or liquid, i.e., the material volume compressibility is positive. Recently, poroelastic metamaterial architectures have been suggested theoretically that allow for an unusual negative effective static volume compressibility - which appears to be forbidden for reasons of energy conservation at first sight. The challenge in the three-dimensional (3D) fabrication of these blueprints lies in the necessary many hollow 3D crosses sealed by thin membranes, which we realize in this work by using 3D laser microlithography combined with a serendipitous mechanism. By using optical-microscopy crosscorrelation analysis, we determine an extraordinarily large negative metamaterial effective volume compressibility of $\kappa_{\text {eff }}=-0.8 \% \mathrm{bar}^{-1}=-80 \mathrm{GPa}^{-1}$ under pressure control.
\end{abstract}

DOI: 10.1103/PhysRevX.7.041060

Subject Areas: Acoustics, Mechanics, Metamaterials

\section{INTRODUCTION}

The static volume compressibility $\kappa$ of a material is defined as

$$
\kappa=-\frac{1}{V}\left(\frac{\partial V}{\partial P}\right)_{T}
$$

with the material volume $V$, the hydrostatic pressure $P$ exerted onto it, and the fixed temperature $T$ [1]. The minus sign is convention and appreciates the fact that ordinary materials shrink $(\partial V / \partial P<0)$ when increasing the pressure onto them, leading to positive compressibility, $\kappa>0$.

Is $\kappa<0$ possible, too? The answer is no. Consider a gas at pressure $P$ within a sealed container and the material under investigation at the container bottom. Suppose a small fluctuation slightly increases the gas pressure. Mathematically, the condition $\kappa<0$ means that the volume of the material increases. However, for this volume increase to happen, the material would need to perform work to compress the gas around it. For passive materials containing no additional energy source, this behavior would obviously violate energy conservation and is thus forbidden. This reasoning for bulk materials also holds true for ordinary porous elastic materials (e.g., a sponge), where the gas can penetrate into the poroelastic structure.

Negative compressibility has to be distinguished from negative incremental $[2,3]$ compressibility for which the

*jingyuan.qu@kit.edu

Published by the American Physical Society under the terms of the Creative Commons Attribution 4.0 International license. Further distribution of this work must maintain attribution to the author(s) and the published article's title, journal citation, and DOI. derivative $\partial V / \partial P$ is only negative in a certain interval of volumes, but positive otherwise. Negative incremental bulk modulus (the inverse of the compressibility) has, e.g., been observed in certain foams under volume control [2]. Under pressure control, such configurations are unstable [3].

A negative static compressibility must also be distinguished from a negative dynamic compressibility. For example, for conditions of an acoustic pressure wave, a negative effective dynamic compressibility simply means that the small time-harmonically varying density and pressure differences on top of large constant offsets are 180 degrees out of phase with respect to each other. Such behavior can occur for frequencies above a resonance inside of the metamaterial unit cells [4-9].

Negative static three-dimensional volume compressibility [10] must be distinguished from negative linear compressibility [11-13]. This notion refers to highly anisotropic structures that expand along one direction upon a hydrostatic pressure increase, while they shrink along the other two directions, or at least along one other direction. This leads to an overall volume decrease [11-13], and hence to a positive volume compressibility, but to negative linear compressibility along one or two axes. Nevertheless, this unusual special class of counterintuitive materials has attracted considerable interest because of its rarity and its potential applications in artificial muscles and actuators [13].

In this paper, following the blueprint of our recent theoretical prediction [14], we fabricate and characterize microstructured metamaterials with a cubic translational lattice that isotropically expand in all three dimensions in response to an increase of the surrounding quasistatic air pressure. This behavior corresponds to a negative effective volume compressibility $\kappa_{\text {eff }}$, which (for reasons given 
below) does not violate the stability and energy reasoning outlined above.

What are possible implications and applications of such unusual behavior? Tailoring the effective compressibility from positive to negative includes the special case of zero compressibility. Such a metamaterial would not change its effective volume when being brought from ambient air to the vacuum of outer space or to the huge hydrostatic pressures of the deep sea-while being made out of perfectly ordinary constituents. The opposite limit of extremely large negative volume compressibility is potentially useful for novel interferometric pressure sensors [11]. Unusually large values of $\left|\kappa_{\text {eff }}\right|$ could also be useful as an actuation mechanism. Materials with negative compressibility might also exhibit interesting properties in regard to three-dimensional acoustic wave propagation. For example, bits of such metamaterials as inclusions within an ordinary gas or liquid should lead to ultralow effective pressure-wave velocities. Combined with some small background absorption, these slow velocities from zero frequency onwards might lead to large absorption coefficients, which are useful for sound absorption.

\section{OPERATION PRINCIPLE}

In our present experimental work, we start from the metamaterial blueprint shown in Fig. 1 [14]. If the surrounding pressure is larger than the pressure inside of the concealed volumes in the eight three-dimensional (3D) crosses within one metamaterial unit cell, the six thin membranes of each cross warp inwards. The lever arms attached to the warping membranes lead to a rotation of the crosses [14]. In any one direction, the two adjacent crosses rotate in opposite directions, such that their rotations cancel and a pure expansion results in this direction. Thus, for isotropic expansion in three dimensions, $2^{3}=8$ crosses are required in each unit cell. The blueprint shown in Fig. 1 [14] has an effective Poisson's ratio of $\nu_{\text {eff }}=-0.97$ and is loosely related to designs [15] used in recently presented experiments on metamaterials with a negative thermal expansion coefficient from positive constituents [16]. It is also loosely related to previous theoretical designs of negative-compressibility auxetic metamaterials based on two different elastic constituents [17]. However, the use of hollow concealed volumes in our blueprint takes advantage of the huge ratio between the compressibility of air and that of the polymer. This large material contrast allows for changing the sign of the quantity

$$
\kappa_{\text {eff }}=-\frac{1}{V_{\text {eff }}}\left(\frac{\partial V_{\text {eff }}}{\partial P}\right)_{T},
$$

which has the same form and unit as the compressibility in (1) with the volume $V$ replaced by the effective volume $V_{\text {eff }}$. We refer to this quantity as the effective compressibility [14]. In the literature, it has sometimes also been referred to (a)
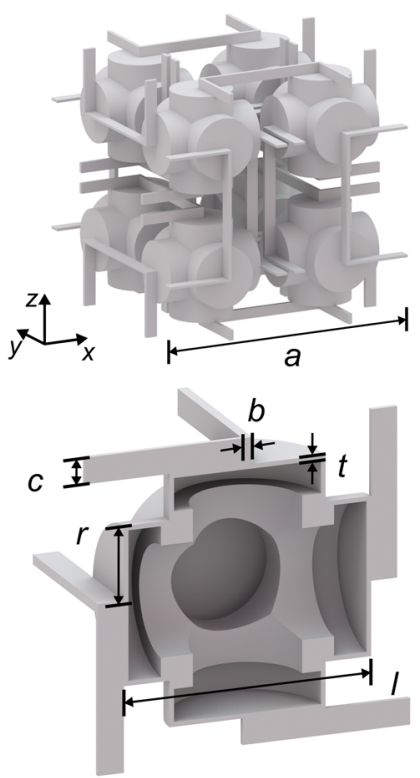

(b)

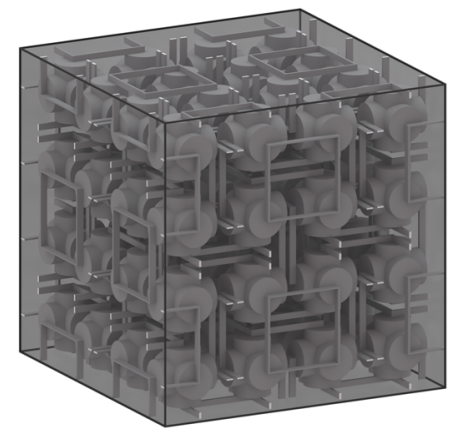

FIG. 1. (a) Blueprint of a (nonprimitive) unit cell of the threedimensional (3D) metamaterial. This unit cell includes eight hollow 3D crosses. The inset shows a cut-open hollow 3D cross. The relevant geometrical parameters are indicated. (b) This unit cell is placed onto a simple-cubic translational lattice with lattice constant $a$, leading to the shown artificial crystal. Its effective volume $V_{\text {eff }}$ is defined by the volume enclosed by the metamaterial's six surface facets (light gray) and exhibits a negative effective static volume compressibility $\kappa_{\text {eff }}$.

as unjacketed compressibility [18]; as compressibility [17] (i.e., no additional adjective); or as net volume compressibility [19]. Altogether, at least four different notions have been used. Following our own previous work [14], we use the notion "effective compressibility" because all metamaterial properties are effective properties.

The energy and stability arguments outlined in the introduction apply to the compressibility $\kappa$, which refers to the volume $V$ enclosed by the constituent material. The hydrostatic pressure $P$ leads to local forces along the local surface normal of the volume $V$ (but not $V_{\text {eff }}$ ). However, $V$ is not directly observable for a porous structure. In contrast, these arguments do not apply to the effective compressibility $\kappa_{\text {eff }}$, which refers to the effective volume $V_{\text {eff }}$ enclosed by the metamaterial sample facets, which are illustrated by the gray surface in Fig. 1(b). The effective 
volume is what one "sees" when looking at the sample. It is important to note that the effective compressibility refers to a part of the combined system of elastic solid and gas. Therefore, it must not be interpreted as the response function of a system, for which causality combined with passivity and stability would demand [20] that the real part of the frequency-dependent response function cannot be negative for zero frequency.

Conceptually, the effective compressibility of the blueprint is unbounded. In the limit of thin membranes, i.e., $t / a \rightarrow 0$, we get $\kappa_{\text {eff }} \rightarrow-\infty$ [14]. In the opposite limit of thick membranes, the effective compressibility eventually converges towards the positive bulk compressibility of the constituent material (the polymer in our case), i.e., $\kappa_{\text {eff }}>0$.

\section{SAMPLE FABRICATION}

In regard to 3D microfabrication of this blueprint, the challenge lies in the making of the hollow 3D crosses, which are concealed by thin membranes. All other parts can immediately be made by standard 3D optical laser lithography (Photonics Professional GT, Nanoscribe $\mathrm{GmbH}$ ) and standard resists (IP-Dip, Nanoscribe $\mathrm{GmbH}$ ). The issue is that, after polymerizing the closed surface of the 3D crosses, the liquid monomer is encapsulated. The established "shell-writing mode" [21] takes advantage of this fact: After the development process, the remaining liquid monomer can be polymerized by ultraviolet-light flood illumination, substantially speeding up the fabrication of large bulk polymer volumes [21]. We have found in the present work, however, that this picture is only correct for sufficiently thick shells or walls. In sharp contrast, for thin shells/walls, the polymer swelling during the development process leads to large stresses, which open up small cracks in the polymer shell/wall through which the liquid monomer escapes and is washed out. Upon drying, the polymer shrinks again and the cracks close. Serendipitously and surprisingly, this process leads to air-sealed individual membranes with about $p \approx 95 \%$ probability (i.e., $1-p^{6} \approx$ $30 \%$ probability of leaking 3D crosses) in our case here-of course, only for a certain range of the membrane thickness $t$ with respect to the lattice constant $a$. Within this range, the enclosed air can only escape via diffusion through the bulk of the thin polymer walls of the final structure on a time scale of some minutes (see below).

The described overall process is somewhat analogous to a syringe needle punching a hole through the soft polymer plug of a bottle and extracting some medication from within. After pulling out the syringe needle, the plug still securely caulks the bottle.

We mention in passing that such a fabrication process may also be of interest for other 3D microstructures incorporating hollow concealed volumes-which have previously been inaccessible for any kind of 3D printing approach.

An example electron micrograph of a fabricated polymer structure composed of $2 \times 2 \times 2=8$ unit cells, i.e., (a)

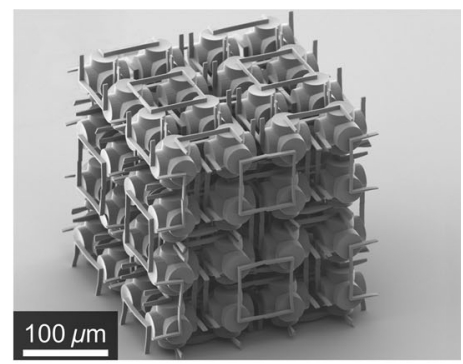

(b)

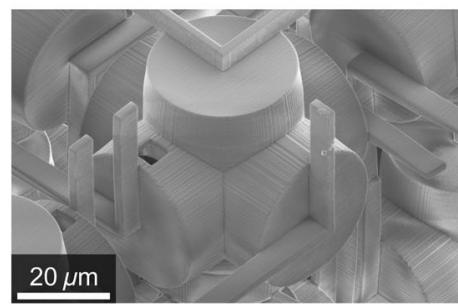

(c)

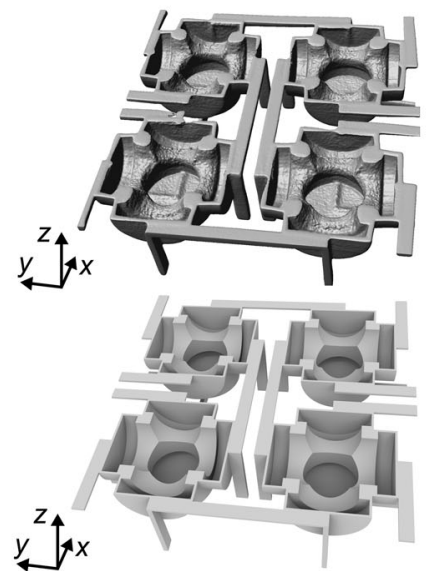

FIG. 2. (a) Electron micrograph of a metamaterial sample composed of $2 \times 2 \times 2=8$ unit cells [see Fig. 1(a)] fabricated by $3 \mathrm{D}$ optical laser lithography. (b) Electron micrograph of a detail of a control structure, in which we have intentionally introduced a hole into each hollow 3D cross, for otherwise identical geometrical parameters. (c) 3D isointensity surface obtained from laser scanning fluorescence microscopy of one unit cell. The shown cut through a part of these fluorescence data reveals that the interior of the hollow 3D crosses is actually hollow. To allow for a direct comparison, the blueprint [also see Fig. 1(a)] is depicted below this measurement for a similar viewing direction.

composed of $8 \times 8=64$ hollow 3D crosses, on glass substrate is depicted in Fig. 2(a) (compare Fig. 1). The target geometrical parameters are $a=150 \mu \mathrm{m}$, $t=1.05 \mu \mathrm{m}, r=18 \mu \mathrm{m}, b=2.25 \mu \mathrm{m}, c=6 \mu \mathrm{m}$, and $l=57 \mu \mathrm{m}$. However, from these images, it cannot be judged whether the 3D crosses are actually hollow. Therefore, we have also inspected the structures by using a laser scanning fluorescence microscope (LSM510 META, with oil-immersion objective Plan-Apochromat $63 \times / 1.40$, Carl Zeiss). Both the liquid monomer and the polymerized material exhibit bright autofluorescence (see, e.g., [22]). Hence, a massive or a filled 3D cross would exhibit bulk fluorescence, whereas a hollow 3D cross 
should exhibit a nonfluorescent interior. Indeed, the cut through the complete 3D data set depicted in Fig. 2(c) shows a hollow interior and a geometry close to the target blueprint, which is also depicted for a similar viewing direction, to allow for direct comparison.

\section{MEASUREMENTS}

To measure the effective metamaterial compressibility, we have built a dedicated optical microscopy setup. It contains a small sample chamber, which can be pressurized with a gas up to excess pressures of about $\Delta P=+4 \times 10^{5} \mathrm{~Pa}$ between the inside and the outside of the chamber. The chamber is mounted on a 3D piezoelectric translation stage, allowing for aligning the sample. The chamber is pressurized by a pressure controller (PQ1, AirCom), connected to an electronic pressure monitor (DC 400, tecsis) and a computer. Within the chamber, the sample can be positioned close to a 1-mm-thick, 1 -cm-diameter fused-silica window, which is glued to the inside of the chamber. This setting allows for a fairly small working distance required for large magnification and good image quality. We use a $20 \times$ objective lens (LD Acroplan $20 \times / 0.40$, Carl Zeiss), a 10-cm focal-length tube lens, and a standard silicon black/white camera (BFLY-PGE-50H5M-C, Point Grey Research), connected to a computer. We have performed such experiments for top views onto the samples as well as for side views. For the latter, the substrate had to be cut close to the metamaterial sample. The acquired images are processed by standard image cross-correlation analysis [23] to derive the displacement-vector field vs surrounding air pressure. From the small relative length change $\Delta L / L \ll 1$, we obtain the relative effective volume change in three dimensions $\Delta V_{\text {eff }} / V_{\text {eff }} \approx 3 \Delta L / L$ and thus

$$
\kappa_{\text {eff }} \approx-3 \frac{\Delta L}{L} \frac{1}{\Delta P} .
$$

Inspecting the displacement-vector field also allows for assessing the homogeneity and the 3D isotropy of the expansion/shrinkage effect (see below).

Results are depicted in Fig. 3. We start from an ambient air pressure of $P=P_{0} \approx 1 \times 10^{5} \mathrm{~Pa}=1$ bar. To minimize leakage and hysteresis effects, we switch to the air pressure $P=P_{0}+\Delta P>P_{0}$ and measure the mean relative length increase $\Delta L / L$ within $2.5 \mathrm{~s}$ and move to the next higher pressure value $P$, etc. Clearly, $\Delta L / L$ as averaged over the $x y$-plane in Fig. 3 increases nearly linearly vs $\Delta P$. The side view of the $x z$-plane reveals that the sample also expands along the $z$ direction (see below). From these observations, we derive an effective volume compressibility of $\kappa_{\text {eff }}=$ $-0.8 \times 10^{-7} \mathrm{~Pa}^{-1}=-0.8 \% \mathrm{bar}^{-1}$. We have reproduced this behavior on five different samples.

As controls, we have repeated the experiments for similar metamaterial samples, however, with intentional holes in the walls of all 3D crosses [see Fig. 2(b)]. In the presence of these holes, a pressure difference between the

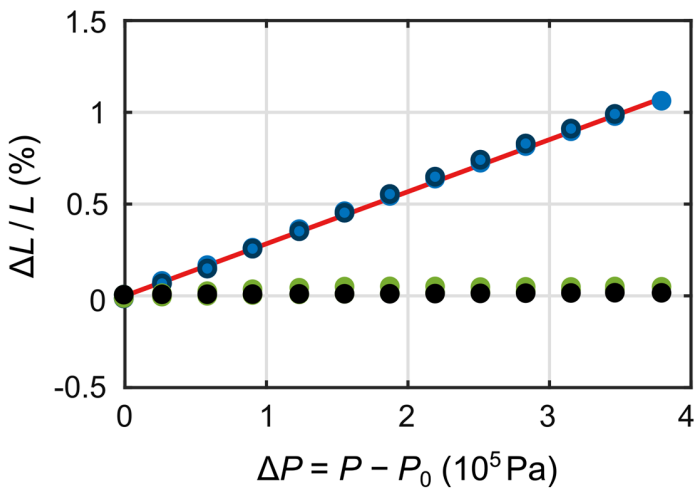

FIG. 3. Measured mean relative length change $\Delta L / L$ vs excess pressure $\Delta P=P-P_{0}>0$, with ambient pressure $P_{0}$. The observed behavior leads to an effective volume compressibility of $\kappa_{\text {eff }}=-0.8 \times 10^{-7} \mathrm{~Pa}^{-1}$. The blue dots are measured (full: pressure ramping up, open: pressure ramping down); the red solid curve is calculated numerically. The green dots correspond to measurements on a control sample, in which we have intentionally introduced a hole in each of the hollow 3D crosses [compare Fig. 2(b)]. The black dots refer to a control experiment on a copper surface.

inside and outside of the 3D crosses cannot build up. As expected on this basis, the resulting relative length changes are much smaller (see green data points in Fig. 3). We ascribe the observed deviations from $\Delta L / L=0$ partly to incorporation of gas molecules into the bulk polymer, leading to a slight swelling of the polymer and hence an increase of the overall structure. Another part of these deviations is a minor artifact of the measurement setup that we will discuss in more detail below.

To investigate the question over which time scale the hollow volumes (without intentional holes) can be considered as sealed, we have performed yet additional experiments shown in Fig. 4. Herein, we increase the air pressure from $P=P_{0}=1 \times 10^{5} \mathrm{~Pa}$ to $P=P_{0}+\Delta P=4.8 \times 10^{5} \mathrm{~Pa}$ in a steplike manner and then monitor the mean $\Delta L / L$ at fixed excess pressure vs time. The sample relaxes towards its original state corresponding to $\Delta L / L=0$ on a time scale of some minutes. We ascribe this time scale to slow diffusion of the air through the polymer membranes. By fitting a single exponential to the experimental data, we derive a time constant of $\tau_{\text {air }}=745 \mathrm{~s}$. There are some minor indications that even better agreement could be obtained by using a double-exponential fit. Two different time constants would be consistent with the fact that air is mainly composed of oxygen (21\%) and nitrogen (78\%). Nitrogen has a smaller gas permeability [24] and hence a larger time constant. It is thus expected to dominate the derived single time constant.

To further solidify our interpretation of gas diffusion through the polymer walls, we have repeated the experiments using the same sample, yet employing carbon dioxide as the pressurizing gas. We find an exponential decay with a much shorter time constant of $\tau_{\mathrm{CO}_{2}}=26 \mathrm{~s}$, 


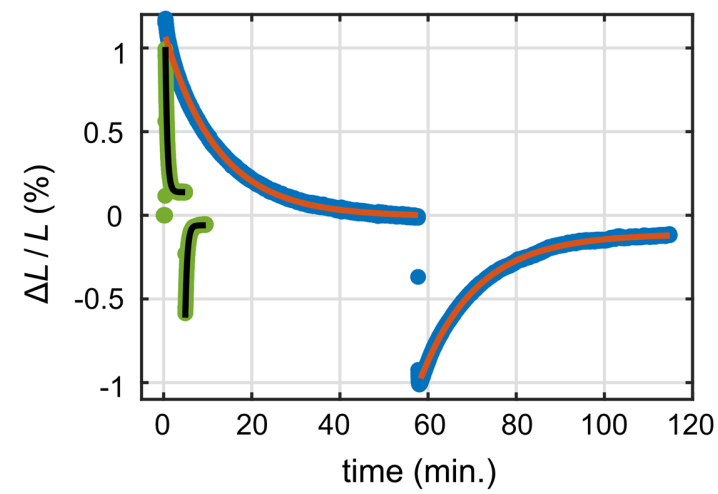

FIG. 4. Measurements as blue dots in Fig. 3. However, at $t=0$, the pressure of the gas is stepwise increased from ambient pressure $P=P_{0}=1.0 \times 10^{5} \mathrm{~Pa}$ to $P=4.8 \times 10^{5} \mathrm{~Pa}$ and then kept constant. The resulting spatial mean $\Delta L / L$ is measured vs time using image cross-correlation analysis. The blue dots correspond to air (mainly composed of oxygen $\mathrm{O}_{2}$ and nitrogen $\mathrm{N}_{2}$ ) as gas, and the green dots to carbon dioxide $\mathrm{CO}_{2}$ instead. In the second half of the cycle, the pressure is switched back down to $P=1.0 \times 10^{5} \mathrm{~Pa}$. The solid curves are single-exponential fits to the experimental data. For air, the derived time constant is $\tau_{\text {air }}=745 \mathrm{~s}$. For carbon dioxide, the exponential time constant is $\tau_{\mathrm{CO}_{2}}=26 \mathrm{~s}$.

followed by a plateau. We ascribe the plateau to swelling of the polymer by incorporation of $\mathrm{CO}_{2}$ into its bulk. For air as surrounding gas, this effect is much smaller as can be seen from the above control experiments on metamaterials with holes in the 3D crosses (see Fig. 3). The observed time constants are roughly compatible with estimates based on literature values of the gas permeabilities through typical polymers [24]. Altogether, these findings confirm that the majority of the hollow 3D crosses are properly sealed and that the gas escapes out of them via diffusion through the bulk of the polymer walls. This behavior has been found on five different samples.

To quantify possible artifacts of the measurement setup itself, we replace the sample by a piece of bulk copper. We can safely assume that copper changes neither its side length nor its volume noticeably for the pressures under investigation. Its small positive compressibility would lead to an extremely small negative ratio $\Delta L / L<0$. Nevertheless, we find an apparent relative length change of $\Delta L / L=+10^{-4}$ at $\Delta P=+3.8 \times 10^{5} \mathrm{~Pa}$ in the recorded optical images (see black dots in Fig. 3). By numerical calculations, we have traced back this artifact to a small outwards warping of the $1-\mathrm{mm}$ thin silica window in response to increasing the pressure inside of the sample chamber. The warping of a dielectric plate within the optical path of the microscope does not induce a lens, but the resulting beam displacements lead to an apparent increase of the object in the camera image plane. Ray-tracing calculations (not shown here) quantitatively reproduce this artifact. Importantly, its magnitude is about two orders of magnitude smaller than the effects for the metamaterial samples (compare blue dots in Fig. 3).

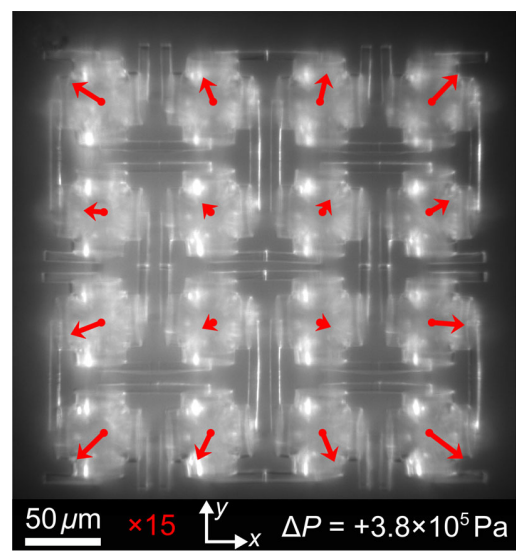

VIDEO 1. Image cross-correlation analysis of a metamaterial sample composed of $2 \times 2 \times 2$ unit cells (compare Fig. 2). Top view onto the $x y$-plane. The displacement vectors shown as red arrows, stretched by a factor of 15 with respect to the black/ white optical microscope image shown in the background, refer to the centers of mass of the unit cells and to a hydrostatic-pressure increase of air from ambient pressure $P=P_{0}=$ $1.0 \times 10^{5} \mathrm{~Pa}$ to $P=P_{0}+\Delta P$, with $\Delta P=3.8 \times 10^{5} \mathrm{~Pa}$. From these arrows, we derive an effective metamaterial volume compressibility of $\kappa_{\text {eff }}=-0.8 \times 10^{-7} \mathrm{~Pa}^{-1}=-0.8 \% \mathrm{bar}^{-1}$.

Therefore, this artifact is quantitatively irrelevant for our conditions. It would be relevant though if samples or aspects exhibiting smaller effects were to be investigated. For example, this artifact prevents us from measuring simultaneously the volume $V$ and hence the ordinary bulk compressibility $\kappa$ of the constituent material according to Eq. (1) along the same lines.

Next, we use the image cross-correlation analysis to investigate the behavior inside of the metamaterial and along the three spatial directions upon increasing the air pressure. Video 1 shows results from a top view onto the $x y$-plane and Video 2 from a side view onto the $x z$-plane. The red arrows exhibit the displacement vectors of the center of mass of the corresponding unit cell. In Video 1, the overall mean displacement vector has been subtracted from all displacement vectors. The length of the arrows grows roughly linearly from the center towards the outside, indicating a homogeneous behavior. Edge effects have only a minor effect. All arrows point roughly radially outwards, indicating an isotropic behavior in the $x y$-plane. Video 2 shows the behavior in the $x z$-plane. Here, we have subtracted the mean of the displacement vectors at the substrate plane at $z=0$ from all displacement vectors. From this view, we do observe edge effects at the substrate, onto which the metamaterial is fixed; hence, its bottom obviously cannot move along the $x$ and $y$ directions near $z=0$. However, the second lattice constant (i.e., layers three and four) already behaves as bulk.

\section{COMPARISON TO THEORY}

Finally, we compare the experimental data with theory, i.e., with numerical finite-element calculations of the 


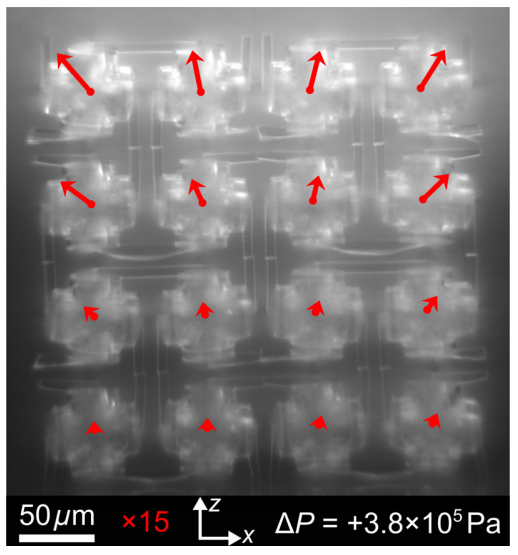

VIDEO 2. Image cross-correlation analysis of a metamaterial sample composed of $2 \times 2 \times 2$ unit cells (compare Fig. 2). Same as Video 1 , but a side view onto the $x z$-plane. The displacement vectors at the substrate plane are set to zero. For the control structure with intentional holes in each of the 3D crosses [compare Fig. 2(b)], the displacement vectors are smaller than the full red dots in the middle of the 3D crosses for both the top view in Video 1 and side view in this Video.

continuum-mechanics equations for the geometry shown in Fig. 1, closely following along the lines of Ref. [14]. To compute bulk behavior, we use periodic boundary conditions along all three spatial directions. We neglect geometric nonlinearities, which is appropriate in the limit $\Delta L / L \ll 1$. Further details have been described in Ref. [14]. The calculated result shown as the red curve in Fig. 3 can directly be compared with the measured data (blue dots). Here, we have chosen the following geometrical parameters [compare Fig. 1(a)]: $t / a=1.15 \%$, $r / a=12 \%, b / a=1.5 \%, c / a=4 \%$, and $l / a=38 \%$ (compare Fig. 1). Theoretically, the relative membrane thickness $t / a$ is the most sensitive parameter [14]. Experimentally, it is the most difficult to determine. In addition, as discussed above, some membranes are leaky. Therefore, we have used $t / a$ as a fit parameter. The quoted values are consistent with the sample electron micrographs within the experimental errors. Furthermore, we have used for the constituent polymer a Young's modulus of $\mathrm{E}=3 \mathrm{GPa}$ and a Poisson's ratio of $\nu=0.4$. Obviously, experiment (blue dots) and theory (red curve) in Fig. 3 agree well, in line with our overall interpretation. From the calculations, we also infer that the observed behavior corresponds to a bulk property; i.e., a metamaterial crystal built from many unit cells behaves similarly to one with only a few unit cells and even more similarly to one comprising only a single unrestricted unit cell.

Figure 5 shows a comparison of experiment and theory upon varying the membrane thickness with respect to that in Fig. 3 over the range accessible to our fabrication approach. As expected from theory, the modulus of the (negative) effective compressibility increases with decreasing membrane thickness.

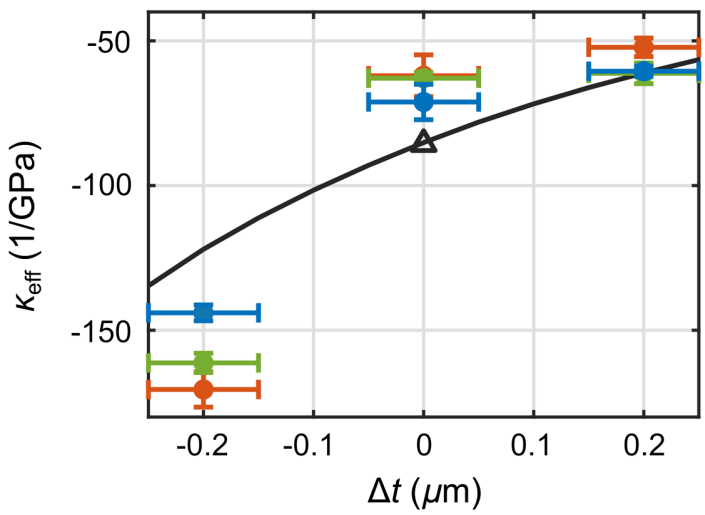

FIG. 5. Effective compressibility vs difference in membrane thickness, $\Delta t$, with respect to the thickness of $t=1.725 \mu \mathrm{m}$ (equivalent to $t / a=1.15 \%$ for $a=150 \mu \mathrm{m}$ ) in Fig. 3. The solid curve is calculated for otherwise identical parameters as the calculations in Fig. 3; the full dots are measured. The different colors correspond to different nominally identical samples. The vertical error bars are \pm three times the standard deviation of the mean for ten measurements each, such as the one shown in Fig. 3, which corresponds to the single open triangle at $\Delta t=0$. The horizontal error bars are estimated. The effective compressibility changes by about a factor of 3 upon changing the membrane thickness by $0.4 \mu \mathrm{m}$.

\section{CONCLUSION}

In conclusion, we have demonstrated experimentally, for what we believe is the first time, a metamaterial with a negative effective compressibility under quasistatic (rather than time-harmonic) conditions. We hope that this advance enables some of the unusual applications and perspectives mentioned above.

\section{ACKNOWLEDGMENTS}

We thank Johann Westhauser (KIT) for technical assistance in the construction of the dedicated measurement setup, Martin Bastmeyer (KIT) for providing us with access to his group's laser scanning confocal fluorescence microscope, and Peter Gumbsch (KIT) for discussions. We acknowledge support by the Helmholtz program Science and Technology of Nanosystems (STN), the Karlsruhe School of Optics \& Photonics (KSOP), the Hector Fellow Academy, the KIT Nanostructure Service Laboratory (NSL), and the Labex ACTION program (Contract No. ANR-11-LABX-0001-01). We also acknowledge support by Deutsche Forschungsgemeinschaft (DFG) and the Open Access Publishing Fund of KIT and the French "Investissements d'Avenir" program, project ISITE-BFC (contract ANR-15-IDEX-03).

[1] A. Sommerfeld, Mechanics of Deformable Bodies: Lectures on Theoretical Physics, Vol. 2 (Academic Press, New York, USA, 1950). 
[2] B. Moore, T. Jaglinski, D. S. Stone, and R. S. Lakes, Negative Incremental Bulk Modulus in Foams, Philos. Mag. Lett. 86, 651 (2006).

[3] R. Lakes and K. W. Wojciechowski, Negative Compressibility, Negative Poisson's Ratio, and Stability, Phys. Status Solidi B 245, 545 (2008).

[4] Z. Liu, X. Zhang, Y. Mao, Y. Y. Zhu, Z. Yang, C. T. Chan, and P. Sheng, Locally Resonant Sonic Materials, Science 289, 1734 (2000).

[5] N. Fang, D. Xi, J. Xu, M. Ambati, W. Srituravanich, C. Sun, and X. Zhang, Ultrasonic Metamaterials with Negative Modulus, Nat. Mater. 5, 452 (2006).

[6] Y. Wu, Y. Lai, and Z.-Q. Zhang, Elastic Metamaterials with Simultaneously Negative Effective Shear Modulus and Mass Density, Phys. Rev. Lett. 107, 105506 (2011).

[7] R. V. Craster and S. Guenneau, Acoustic Metamaterials: Negative Refraction, Imaging, Lensing and Cloaking (Springer Science \& Business Media, New York, 2012), Vol. 166.

[8] M. Kadic, T. Bückmann, R. Schittny, and M. Wegener, Metamaterials Beyond Electromagnetism, Rep. Prog. Phys. 76, 126501 (2013).

[9] S. A. Cummer, J. Christensen, and A. Alù, Controlling Sound with Acoustic Metamaterials, Nat. Rev. Mater. 1, 16001 (2016).

[10] Z. G. Nicolaou and A. E. Motter, Mechanical Metamaterials with Negative Compressibility Transitions, Nat. Mater. 11, 608 (2012).

[11] R. H. Baughman, S. Stafström, C. Cui, and S. O. Dantas, Materials with Negative Compressibilities in One or More Dimensions, Science 279, 1522 (1998).

[12] J. N. Grima, D. Attard, R. Caruana-Gauci, and R. Gatt, Negative Linear Compressibility of Hexagonal Honeycombs and Related Systems, Scr. Mater. 65, 565 (2011).

[13] A. B. Cairns, J. Catafesta, C. Levelut, J. Rouquette, A. Van Der Lee, L. Peters, A. L. Thompson, V. Dmitriev, J. Haines, and A. L. Goodwin, Giant Negative Linear Compressibility in Zinc Dicyanoaurate, Nat. Mater. 12, 212 (2013).

[14] J. Qu, M. Kadic, and M. Wegener, Poroelastic Metamaterials with Negative Effective Static Compressibility, Appl. Phys. Lett. 110, 171901 (2017).

[15] G. W. Milton, The Theory of Composites (Cambridge University Press, Cambridge, UK, 2002).

[16] J. Qu, M. Kadic, A. Naber, and M. Wegener, MicroStructured Two-Component 3D Metamaterials with Negative Thermal-Expansion Coefficient from Positive Constituents, Sci. Rep. 7, 40643 (2017).

[17] R. Gatt and J. N. Grima, Negative Compressibility, Phys. Status Solidi RRL 2, 236 (2008).

[18] M. A. Biot and D. G. Willis, The Elastic Coefficients of the Theory of Consolidation, J. Appl. Mech. 24, 594 (1957).

[19] J. N. Grima, D. Attard, and R. Gatt, Truss-Type Systems Exhibiting Negative Compressibility, Phys. Status Solidi B 245, 2405 (2008).

[20] A. B. Pippard, Response and Stability: An Introduction to the Physical Theory (CUP Archive, Cambridge, England, 1985).

[21] M. F. Schumann, S. Wiesendanger, J. C. Goldschmidt, B. Bläsi, K. Bittkau, U. W. Paetzold, A. Sprafke, R. B. Wehrspohn, C. Rockstuhl, and M. Wegener, Cloaked Contact Grids on Solar Cells by Coordinate Transformations: Designs and Prototypes, Optica 2, 850 (2015).

[22] Q. Shi, B. Sontheimer, N. Nikolay, A. W. Schell, J. Fischer, A. Naber, O. Benson, and M. Wegener, Wiring Up Pre-Characterized Single-Photon Emitters by Laser Lithography, Sci. Rep. 6, 31135 (2016).

[23] T. Bückmann, N. Stenger, M. Kadic, J. Kaschke, A. Frölich, T. Kennerknecht, C. Eberl, M. Thiel, and M. Wegener, Tailored 3D Mechanical Metamaterials Made by Dip-in Direct-Laser-Writing Optical Lithography, Adv. Mater. 24, 2710 (2012).

[24] H. Lin and B. D. Freeman, Gas Solubility, Diffusivity and Permeability in Poly(Ethylene Oxide), J. Membr. Sci. 239, 105 (2004). 